\title{
A Kidney Segmentation Framework for Dynamic Contrast Enhanced Magnetic Resonance Imaging
}

\author{
SENIHA E. YUKSEL \\ Department of Computer and Information Science and Engineering, University of Florida, \\ Gainesville, FL 32611, USA (seyuksel@cise.ufl.edu)
}

AYMAN EL-BAZ

ALY A. FARAG

Computer Vision and Image Processing Laboratory, University of Louisville, Louisville, KY 40292,USA(elbaz@cvip.uofl.edu)

\author{
MOHAMED EL-GHAR \\ TAREK ELDIASTY \\ MOHAMED A. GHONEIM \\ Urology and Nephrology Center, Mansoura University, Mansoura, Egypt
}

(Received 26 April 2006; accepted 12 July 2006)

\begin{abstract}
In the United States, more than 12000 renal transplantations are performed annually; but the transplanted kidneys face a number of surgical and medical complications that cause a decrease in their functionality. In an effort to understand the reasons for this functionality decrease, considerable attention has recently been focused on Dynamic Contrast Enhanced Magnetic Resonance Imaging (DCE-MRI) due to its superior functional and anatomical information. The biggest challenge in the analysis of DCE-MRI is the segmentation of kidneys from abdomen images because of the high noise and partial volume effects introduced during the rapid and repeated scanning process. In this paper, a general framework is introduced for the segmentation of kidneys from DCE-MR images of the abdomen. The proposed segmentation algorithm consists of three main steps. In the first step, an average kidney shape is constructed from a dataset of previously segmented kidneys, and an average signed distance map density is obtained describing the shape of the kidneys. In the second step, the gray level density is calculated for a given new kidney image using a modified expectation maximization (EM) algorithm. In the third step, a deformable model is evolved based on the two density functions obtained from the previous two steps: the first one describes the prior shape of the kidney, and the second one describes the distribution of the gray level inside and outside the kidney region. The new deformable model is able to handle intricate shapes without getting stuck in edge points; and gives very promising results that are comparable to radiologists' segmentation.
\end{abstract}

Keywords: Dynamic Contrast Enhanced MRI, Gd-DTPA, acute rejection, renal transplantation, deformable models, segmentation, shape database

\section{INTRODUCTION}

Acute rejection - the immunological response of the human immune system to the foreign kidney - is the most important cause of graft failure after renal transplantation (Rigg 1995), 

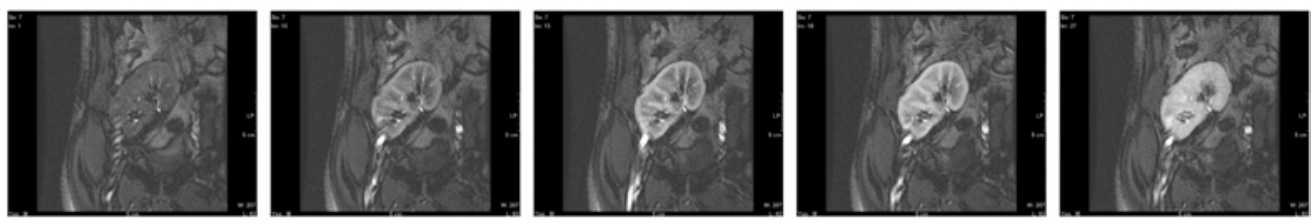

Figure 1. Current protocol. For each subject, 150 abdomen images are taken from one cross-section with 4 second intervals. An example DCE-MRI series for one subject is shown here with five of the images in the sequence, numbered $(1,10,13,18,27)$. Details of the protocol are given in Yuksel (2005).

and the differential diagnosis of acute transplant dysfunction remains a difficult clinical problem. Currently, the diagnosis of rejection is done via biopsy, but biopsy has the downside effect of subjecting the patients to risks like bleeding and infections. Moreover, the relatively small needle biopsies may lead to over or underestimation of the extent of inflammation in the entire graft (Yang et al. 2001). Therefore, a noninvasive and repeatable technique is not only helpful but also necessary in the diagnosis of acute renal rejection.

In Dynamic Contrast Enhanced Magnetic Resonance Imaging (DCE-MRI), a contrast agent called Gd-DTPA is injected into the bloodstream, and as it perfuses into the organ, the kidneys are imaged rapidly and repeatedly. During the perfusion, Gd-DTPA causes a change in the relaxation times of the tissue and creates a contrast change in the images as shown in Figure 1. As a result, the patterns of the contrast change gives functional information, while MRI provides good anatomical information which helps in distinguishing the diseases that affect different parts of the kidneys. However, even with an imaging technique like DCE-MRI, there are several problems: (i) the spatial resolution of the dynamic MR images is low due to fast scanning; (ii) the images suffer from motion induced by the breathing patient, which necessitates advanced registration techniques; (iii) the intensity of the kidney changes non-uniformly as the contrast agent perfuses into the cortex, which complicates the segmentation procedures.

To the best of our knowledge, there has been only limited work on dynamic MRI to overcome the problems of registration and segmentation. For the registration problem, Gerig et al. (1992) proposed using the Hough transform to register the edges in an image to the edges of a mask, and Giele (2002) introduced a phase difference movement detection method to correct kidney displacements. Both of these studies required building a mask manually by drawing the kidney contour on a 2D DCE-MRI image, followed by registration of the time frames to this mask. Therefore, the inherent segmentation (by drawing the contours) was essentially manual. For automatic registration that does not depend on segmentation, we previously proposed using mutual information registration (Yuksel 2005, Yuksel et al. 2005).

For the segmentation problem, Boykov et al. (2001) described the use of graph cuts using Markov models, in which the energy is minimized depending on the manually exerted seed points. Giele (2002) used image subtraction to obtain a mask, and closed the possible gaps using a hull function. For further segmenting the medulla and the cortex structures, repeated erosions were applied to the mask to obtain several rings; however, in such rings, the 
medulla structures were intermixed with the cortex structures, so a correlation study had to be applied to better classify the cortical and medullary pixels.

Following these studies, a multi-step approach to segmentation and registration was introduced by Sun et al. (2004). Initially, the edges are aligned using an image gradient based similarity measure considering only translational motion. Once roughly aligned, a highcontrast image is subtracted from a pre-contrast image to obtain a kidney contour; which is then propagated over the other frames searching for the rigid registration parameters. For segmentation of the cortex and medulla, a level sets approach was used.

Most of these efforts used healthy transplants in the image analysis, and edge detection algorithms were sufficient. However; in the case of acute rejection patients, the uptake of the contrast agent is decreased, so edge detection fails to give connected contours. For this reason, we found the combined use of gray level and prior shape information gave better results.

\section{SEGMENTATION FRAMEWORK}

Within our segmentation framework, the user is asked to select an early enhancement image (typically the 5-15th images of the sequence) and a late enhancement image (typically the 30-40th images of the sequence). In an early enhancement image, the cortical and medullary tissue are distinguishable; whereas in a late enhancement image, the contrast is increased all over the kidney, easing the segmentation process. Thus, a two-stage process is used for segmentation in which (i) the kidney is segmented from the surrounding structures using the late-enhancement image, and (ii) the segmented kidney is further segmented into cortex and medulla using the early-enhancement image, as illustrated in Figure 2.

In segmenting the kidney from the surrounding structures, the gray level distribution of the kidney and surrounding organs is not highly distinguishable; thus, we developed a deformable model which takes into account both the gray level distribution of the late-enhanced kidney and a shape model of the kidney that depends on a sign distance map. The gray level distribution is estimated using our modified EM algorithm (Farag et al. 2004), which uses a linear combination of Gaussians with positive and negative components.

The rest of the paper is organized as follows: in the next section, we describe our modified EM algorithm using linear combination of Gaussian distribution with positive and negative components. In Section 2.2, we describe how to obtain a shape model from a database of formerly segmented images. From these images, we calculate the signed distance maps and estimate their density. By representing both shape and intensity as 1-D density functions, we obtain a fast and computationally inexpensive deformable model which is described in Section 2.3. Results and further directions are discussed in Section 3.

\subsection{Density Estimation with a Modified EM Algorithm}

Our modified Expectation-Maximization algorithm approximates an empirical probability density function of scalar data with a linear combination of discrete Gaussians (LCDG) with positive and negative components. Due to both positive and negative components, the LCDG approximates inter-class transitions more accurately than a conventional mixture of only 


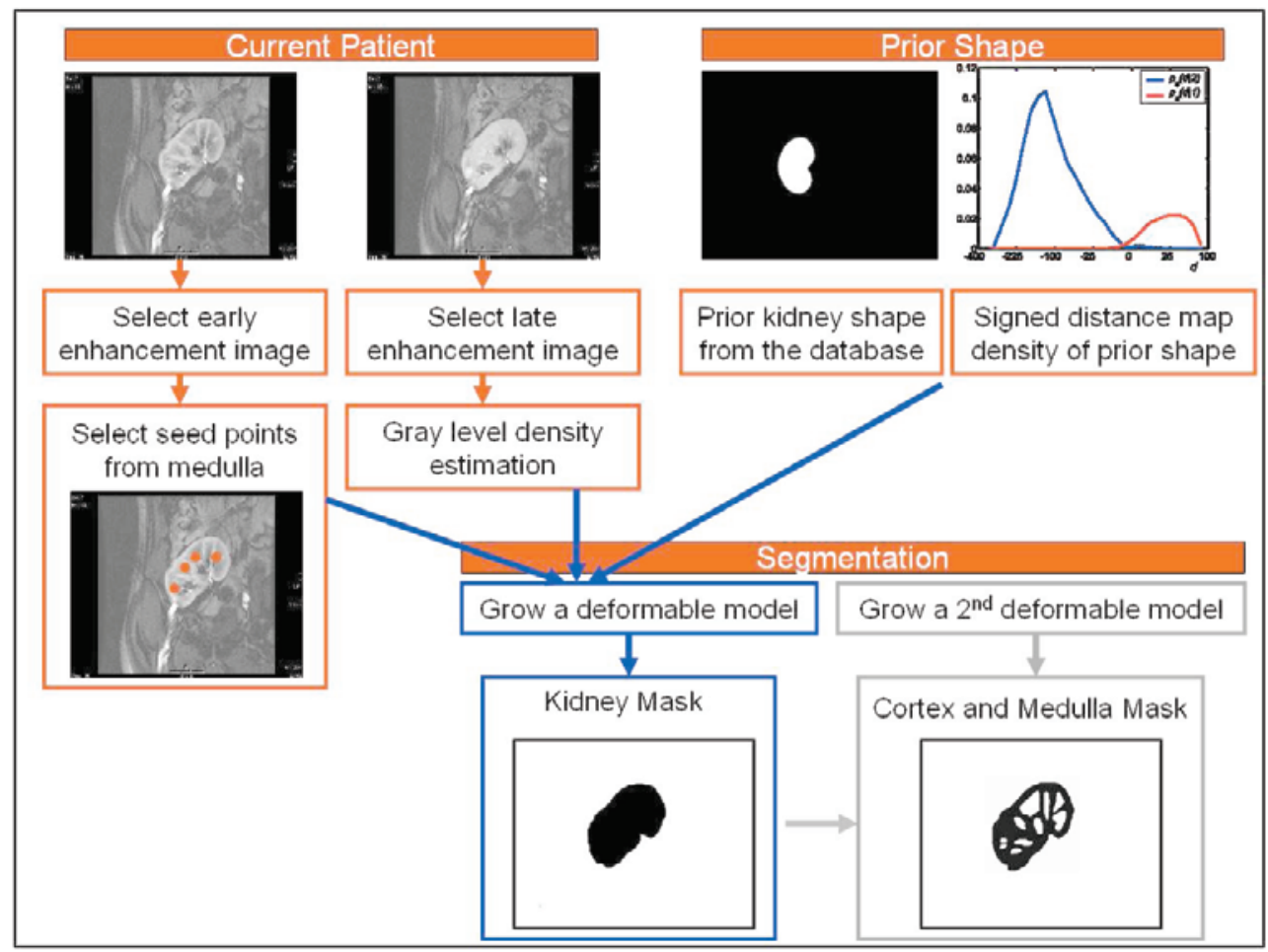

Figure 2. Segmentation framework. An early enhancement image, seed points, and a late enhancement image are selected from the current patient. For kidney segmentation, a deformable model is initialized at the seed points to evolve with the gray level density estimation and the signed distance map density estimation of prior shape. With a second deformable model, cortex and medulla are extracted from the kidney region.

positive Gaussians. In the following, we describe this model to estimate the marginal density for the gray level distribution $p_{g}(q)$ in each region where $q$ is the gray level. The same approach is also used to estimate the density of the signed distances $p_{s}(d)$ in the given image where $d$ is the signed distance inside and outside the object.

Let $q \in \mathbf{Q}=\{0,1, \ldots, Q-1\}$ denote the $Q$-ary gray level. The discrete Gaussian (DG) is defined as the discrete probability distribution $\Psi_{\theta}=(\psi(q \mid \theta): q \in \mathbf{Q})$ on $\mathbf{Q}$ such that

$$
\begin{aligned}
& \psi(q \mid \theta)=\Phi_{\theta}(q+0.5)-\Phi_{\theta}(q-0.5) \quad \text { for } \quad q=1, \ldots, Q-2, \\
& \psi(0 \mid \theta)=\Phi_{\theta}(0.5), \quad \psi(Q-1 \mid \theta)=1-\Phi_{\theta}(Q-1.5)
\end{aligned}
$$

where $\Phi_{\theta}(q)$ is the cumulative Gaussian (normal) probability function with a shorthand notation $\theta=\left(\mu, \sigma^{2}\right)$ for its mean, $\mu$, and variance, $\sigma^{2}$.

In contrast to a conventional mixture of Gaussians and/or other simple distributions, one per region, we closely approximate the empirical gray level distribution for the given image with a LCDG having $C_{\mathrm{p}}$ positive and $C_{\mathrm{n}}$ negative components: 


$$
p_{g: \mathbf{w}, \Theta}(q)=\sum_{r=1}^{C_{\mathrm{p}}} w_{\mathrm{p}, r} \psi\left(q \mid \theta_{\mathrm{p}, r}\right)-\sum_{l=1}^{C_{\mathrm{n}}} w_{\mathrm{n}, l} \psi\left(q \mid \theta_{\mathrm{n}, l}\right)
$$

under the obvious restriction on the weights $\mathbf{w}=\left[w_{p, .}, w_{n, .}\right]$ :

$$
\sum_{r=1}^{C_{\mathrm{p}}} w_{\mathrm{p}, r}-\sum_{l=1}^{C_{\mathrm{n}}} w_{\mathrm{n}, l}=1 .
$$

To estimate the parameters for the model shown in equation (2), we modify the conventional EM algorithm to take into account both positive and negative discrete Gaussian components. Our modified EM algorithm for LCDG maximizes the log-likelihood of the empirical data to find the optimal model parameters assuming statistical independence of the mixed signals:

$$
L(\mathbf{w}, \boldsymbol{\Theta})=\sum_{q \in \mathbf{Q}} f(q) \log p_{\mathbf{w}, \Theta}(q) .
$$

A local maximum of the log-likelihood in equation (3) is given with the EM process extending the one in Schlesinger and Hlavac (2002) onto alternating signs of the components. Let

$$
p_{\mathbf{w}, \Theta}^{[m]}(q)=\sum_{r=1}^{C_{\mathrm{p}}} w_{\mathrm{p}, r}^{[m]} \psi\left(q \mid \theta_{\mathrm{p}, r}^{[m]}\right)-\sum_{l=1}^{C_{\mathrm{n}}} w_{\mathrm{n}, l}^{[m]} \psi\left(q \mid \theta_{\mathrm{n}, l}^{[m]}\right)
$$

denote the current LCDG at iteration $m$. Relative contributions of each signal $q \in \mathbf{Q}$ to each positive and negative DG at iteration $m$ are specified by the respective conditional weights

$$
\pi_{\mathrm{p}}^{[m]}(r \mid q)=\frac{w_{\mathrm{p}, r}^{[m]} \psi\left(q \mid \theta_{\mathrm{p}, r}^{[m]}\right)}{p_{\mathbf{w}, \Theta}^{[m]}(q)}, \quad \pi_{\mathrm{n}}^{[m]}(l \mid q)=\frac{w_{\mathrm{n}, l}^{[m]} \psi\left(q \mid \theta_{\mathrm{n}, l}^{[m]}\right)}{p_{\mathbf{w}, \Theta}^{[m]}(q)}
$$

such that the following constraints hold:

$$
\sum_{r=1}^{C_{\mathrm{p}}} \pi_{\mathrm{p}}^{[m]}(r \mid q)-\sum_{l=1}^{C_{\mathrm{n}}} \pi_{\mathrm{n}}^{[m]}(l \mid q)=1, \quad q=0, \ldots, Q-1 .
$$

The following two steps iterate until the log-likelihood changes become small:

E- step $[m+1]$ : Find conditional expectations of the parameters $\mathbf{w}^{[m+1]}, \boldsymbol{\Theta}^{[m+1]}$ using the fixed weights of equation (4) for the step $m$ as conditional probabilities, and

M- step $[m+1]$ : Find the latter weights by maximizing $L(\mathbf{w}, \boldsymbol{\Theta})$ under the fixed parameters $\mathbf{w}^{[m+1]}, \boldsymbol{\Theta}^{[m+1]}$.

As shown in Schlesinger and Hlavac (2002), this block relaxation process converges to a local maximum of the likelihood in equation (3). The modified EM algorithm is sensi- 


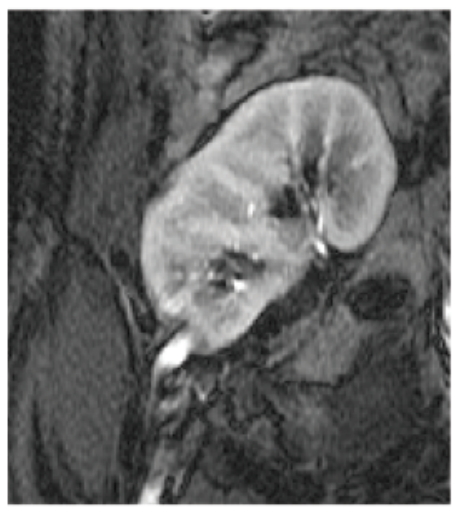

(a)

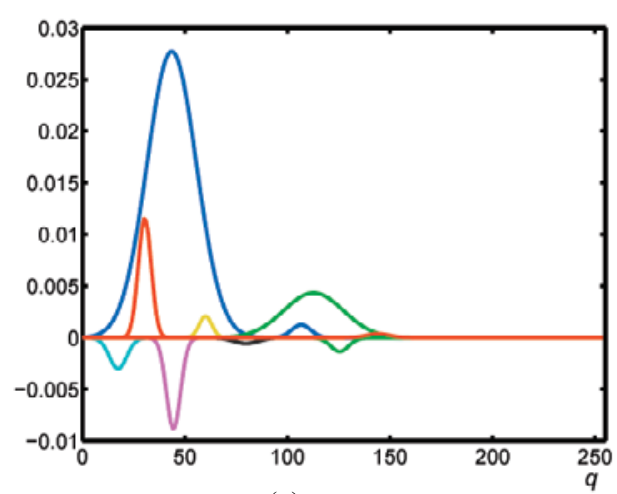

(c)

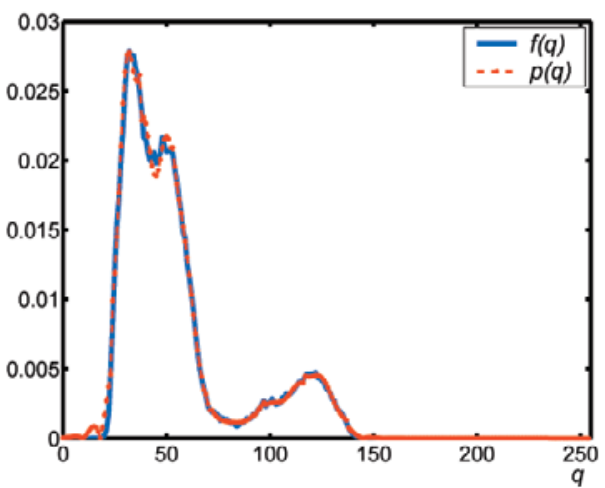

(b)

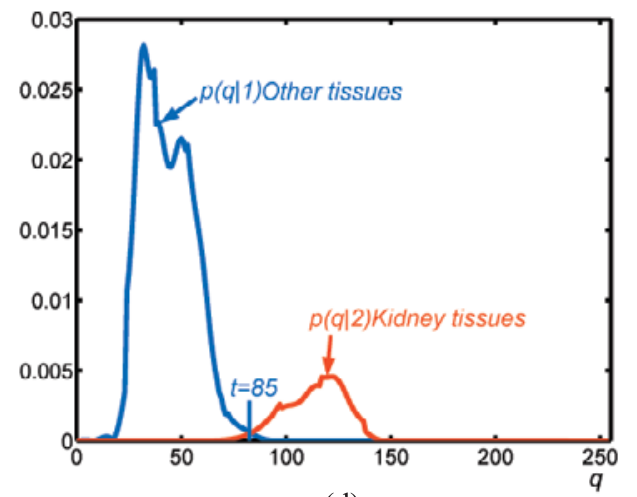

(d)

Figure 3. An MRI scan of a kidney (a), and its gray level density estimation with the Modified EM Algorithm. (b) LCG components of the density estimation; (c) final estimated density $p_{g}(q)$ for the empirical density $f(q)$ of the kidney image; (d) marginal density estimation for each class.

tive to the initialization, so a sequential EM algorithm has been used to obtain an initialization (Farag et al. 2006). The final mixed LCDG-model $p_{C}(q)$ is partitioned into the $K$ LCDG-submodels $P_{[k]}=[p(q \mid k): q \in \mathbf{Q}]$, one per class, or region $k=1, \ldots, K$, by associating subordinate discrete Gaussians with the dominant terms so that the expected misclassification rate is minimized. From the sequence given in Figure 2, a scan of a kidney is given in Figure 3(a), and the steps to estimate its gray level density are illustrated in Figure 3(b, c, d).

\subsection{Forming the Shape Database}

To obtain a prior shape model for the kidney, we used a database of 30 subjects, 5 of which are shown in Figure 4(a). As a first step, we manually segmented the kidneys from these subjects and aligned them using affine registration (translation, rotation and scaling) as shown in Figure 4(b). Then we obtained the 2D shape model of a kidney with the following steps: 

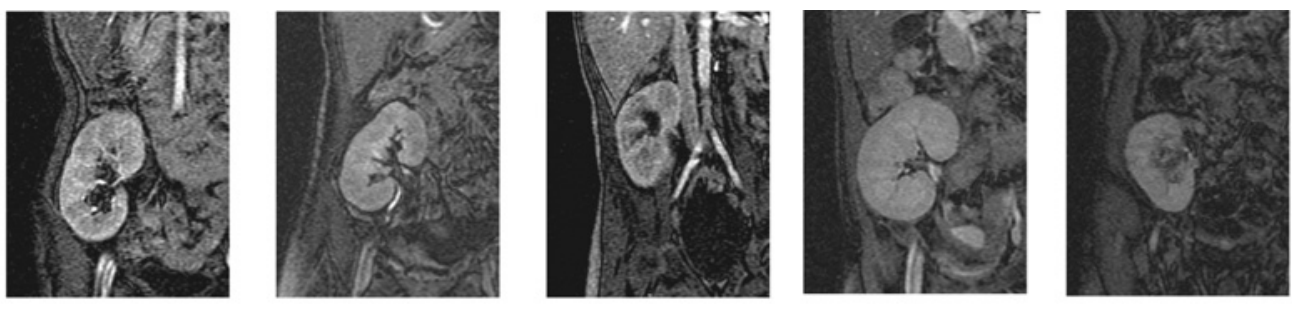

(a)
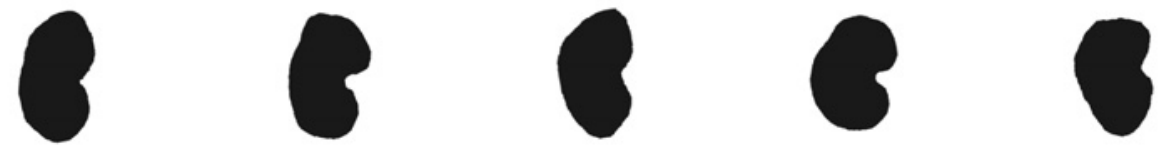

(b)
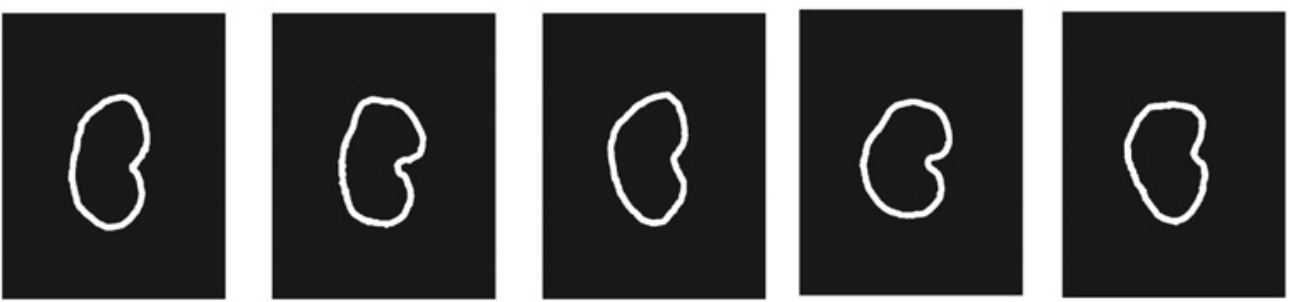

(c)
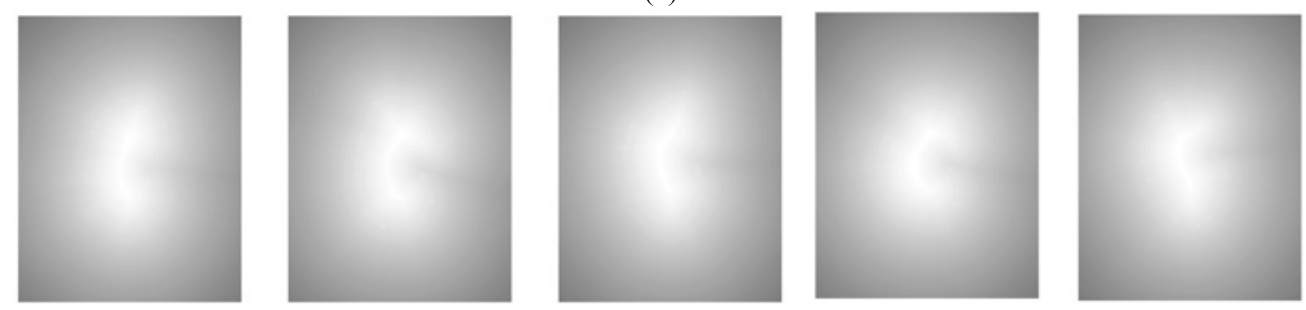

(d)
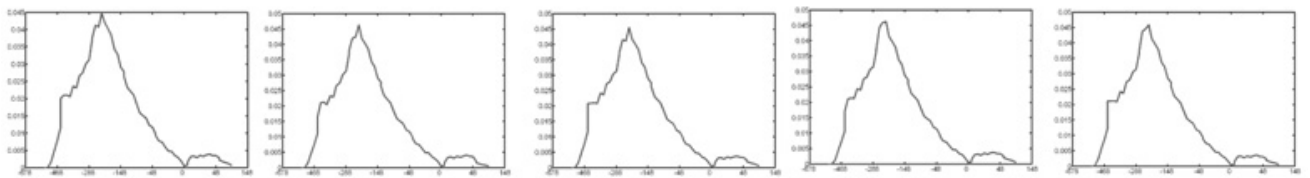

(e)

Figure 4. (a) A sample of the kidney database from five subjects. (b) Affine registration of the data set. (c) Boundaries of the aligned kidneys. (d) Signed distance maps of the data set representing the distances to the kidney contours. (e) Signed distance map densities obtained from (d) using the modified EM algorithm. 
1. Manually segment the kidneys from the surrounding organs and align the segmented data set using affine registration. The results of affine registration is shown in Figure 4(b).

2. From the database given in Figure 4(a), find the 2D edge $V$ of the kidneys as shown in Figure 4(c).

3. For each edge image, calculate the signed distance function $S_{d}(i, j)$ as shown in Figure 4(d). Signed distance function basically calculates the Euclidean distance between a point on the image and the closest point to it on the contour, and gives a positive distance value if the point on the image is inside the object, or gives a negative distance otherwise. In mathematical terms, signed distance function $S_{d}(i, j)$ can be represented as:

$$
S_{d}(i, j)= \begin{cases}0 & (i, j) \in V \\ d((i, j), V) & (i, j) \in R_{V} \\ -d((i, j), V) & \text { Otherwise }\end{cases}
$$

where $R_{V}$ is the region which lies inside the shape and $d((i, j), V)$ is the minimum Euclidean distance between the image location $(i, j)$ and the curve $V$. This representation is invariant to translation and rotation.

4. Calculate the occurrences of distance values (i.e. generate distance map histograms) for all the database, and estimate their density with the modified EM algorithm. These densities are shown in Figure 4(e).

5. Add the densities found in the previous step and obtain an average density function, as shown in Figure 5(a).

6. Average the signed distance values calculated in Step 3. If average signed distance $S_{a v}(i, j)$ of a pixel $(i, j)$ is positive, assign the pixel to be kidney, otherwise make it a background in the average shape image. With this step, an average kidney as shown in Figure 5(f) is obtained. It is worth noting the similarity of this average kidney to the kidney images found in anatomy books.

With these steps, shape modelling is completed, and an average kidney shape, as well as an average signed distance density, is obtained. These are the only two things that are kept and used in the deformable model formulation; i.e. the average density function (Figure 5(e)) and the average kidney shape (Figure 5(f)). Any new kidney image that needs to be segmented is first registered to this average kidney shape, then the average signed distance density is used in the deformable contour energy calculations.

\subsection{Deformable Model}

In conventional deformable models, surfaces move in the direction that minimizes an energy function that is composed of internal and external energy components given as:

$$
E=E_{\text {int }}+E_{\text {ext }}=\int_{\tau \in T}\left(\xi_{\text {int }}(\phi(\tau))+\xi_{\text {ext }}(\phi(\tau))\right) \mathrm{d} \tau
$$

where $\xi_{\text {int }}(\phi(\tau))$ and $\xi_{\text {ext }}(\phi(\tau))$ denote the internal and external force, respectively. 


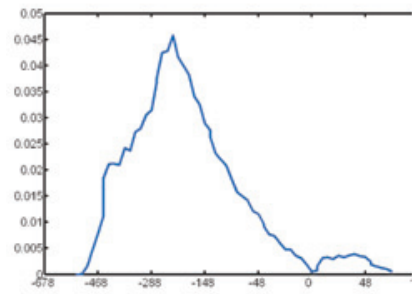

(a)

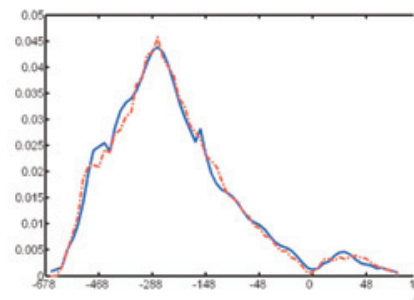

(d)

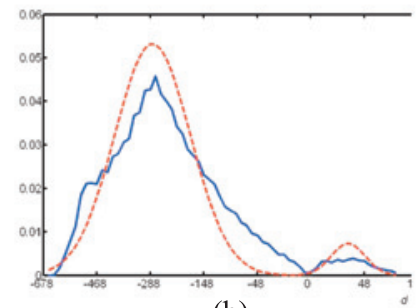

(b)

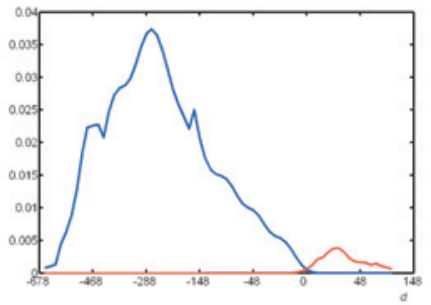

(e)

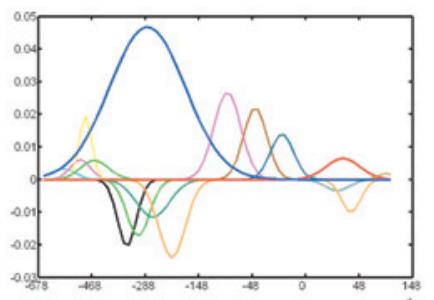

(c)

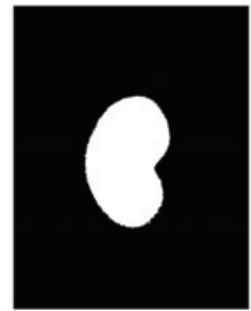

(f)

Figure 5. (a) Average signed distance map density. (b) Estimated two dominant modes which presents the kidney area and its background; (c) positive and negative LCDG components for finer approximation; (d) final density estimation; and (e) densities for two classes (red is for the kidney, blue is for the background). (f) Average kidney shape. Pixels with positive signed distance map values are assigned to be white indicating the kidney region; pixels with negative signed distance map values are assigned to be black, indicating the background.

Typical external forces designed in Witkin et al. (1987) lead a deformable model toward edges in a 2D grayscale image. This and other traditional external forces (e.g. based on lines or, edges, or the gradient vector flow) fail to make the deformable model closely approach an intricate boundary with concavities. As a solution to this problem, we modify the external energy component of this energy formulation, and formulate an energy function using the density estimations of two distributions: the signed distance map from shape models and the gray level distribution. The external energy component of our deformable models is formulated as (El-Baz et al. 2005):

$$
\xi_{\text {ext }}(\phi(\tau))=\left\{\begin{array}{rll}
-p(q \mid k) p_{s}(d \mid k) p(k) & \text { if } & k=k^{*} \\
p(q \mid k) p_{s}(d \mid k) p(k) & \text { if } & k \neq k^{*}
\end{array}\right.
$$

In this formulation, $k$ is the region label with $k=1$ for the background and $k=2$ for kidney, $q$ is the gray level, $d$ is the signed distance; where $p_{g}(q \mid k)$ is the density estimation of the gray level as described in Section 2.1; and $p_{s}(d \mid k)$ represents the density that describes the signed distance map inside and outside the object as calculated in Section 2.2. With this energy function, the stochastic external force for each control point $\phi(\tau)$ of the current deformable model evolves in a region $k^{*}$ as shown in Figure 6(a). 


\section{S.E. YUKSEL ET AL.}

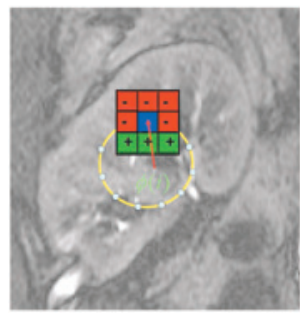

(a)

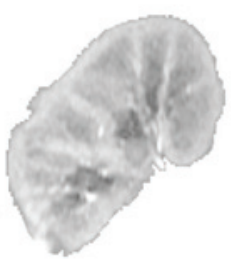

(b)

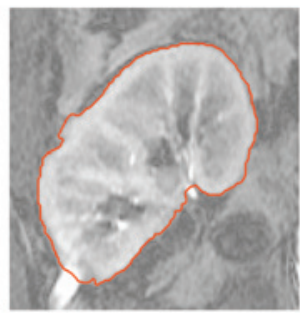

(c)

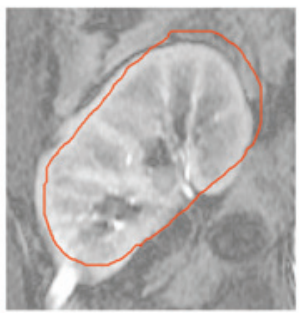

(d)

Figure 6. The proposed deformable model in the kidney (a). For each of the neighbors of the control point $\phi(i)$, four values are calculated to form the external energy: two values from the signed distance density estimation $\left(p_{s}(d \mid k=1)\right.$ and $\left.p_{s}(d \mid k=2)\right)$, and two values from the gray level density estimation $\left(p_{g}(q \mid k=1)\right.$ and $\left.p_{g}(q \mid k=2)\right)$. Radiologist's segmentation (b). Our segmentation results in an error $=0.3820$ w.r.t the radiologist segmentation (c). Segmentation results using the algorithm in Tsai et al. (2003) with error $=10.1(d)$.

For segmentation into cortex and medulla, we first ask the user to select seed points from the possible medulla regions, and we allow separate deformable models to grow in these regions, this time with only the gray level dependent energy function. Therefore, the deformable model successfully moves within a lower and upper threshold to capture the medulla regions, and the rest is the cortex.

\section{RESULTS}

Our segmentation results are comparable to the radiologist's segmentation ( 0.382 for the example given in Figure 6; and outperforms other well-known approaches such as that of Tsai et al. (2003), which gives an error of 10.1. In the proposed approach, after the best image selected by the user is segmented, its shape is used to segment the rest of the sequence as shown in Figure 7(b) w.r.t the radiologist's segmentation given in Figure 7(a). For segmentation into cortex and medulla, the deformable model is evolved with only the gray level information, with results shown in Figure 7(c, d).

In this paper we have presented a framework for the segmentation of kidneys from Dynamic Contrast Enhanced Magnetic Resonance Images, and introduced a new deformable model with an energy as a function of both the gray level density and the prior shape information as 1D density functions. For these density estimations, we introduced a modified EM algorithm. This paper builds upon previous work and presents segmentation as a complete framework in more detail. For more information on other applications of this approach, the reader is referred to Elbaz et al. (2005), to Farag et al. (2006) for more information on our current efforts towards the non-rigid registration of segmented kidneys, and to El-Baz et al. (2006) for an overall picture of the project, which is the design of a CAD system for the diagnosis of kidney diseases. 


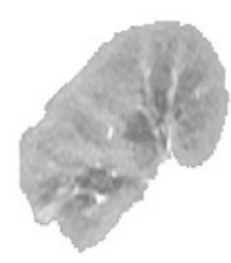

(a)

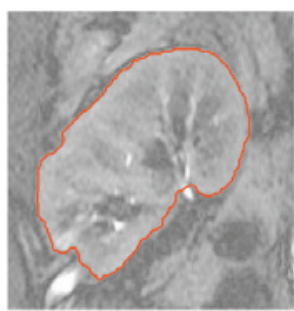

(b)

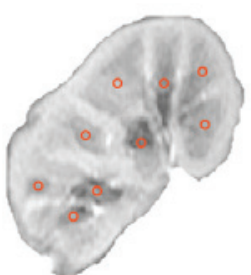

(c)

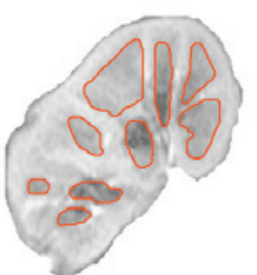

(d)

Figure 7. (a) Radiologist's segmentation for another slice of the same kidney. (b) Our segmentation result using the shape obtained in Figure 6. The error is 0.3997 w.r.t the radiologist's segmentation. (c) Segmentation of the cortex with the proposed algorithm. Several medullary seeds are initialized, and the deformable model is allowed to grow from these seed points based on intensity. (d) The results of the segmentation into cortex and medulla.

\section{REFERENCES}

Boykov, Y., Lee, V. S., Rusinek, H., and Bansal, R., 2001, "Segmentation of dynamic N-D data sets via graph cuts using Markov models," in Proceedings of the 4th International Conference on Medical Image Computing and Computer-Assisted Intervention (MICCAI), Utrecht, The Netherlands, October 14-17, pp. 1058-1066.

El-Baz, A., Yuksel, S. E., Shi, H., Farag, A. A., El-Ghar, M. A., Eldiasty, T., and Ghoneim, M. A., 2005, "2D and 3D shape based segmentation using deformable models," in Proceedings of International Conference on Medical Image Computing and Computer Assisted Intervention (MICCAI), Palm Springs, CA, October 26-29, pp. 821-829.

Farag, A. A., El-Baz, A., and Gimel'farb, G., 2004, "Density estimation using modified expectationmaximization for a linear combination of Gaussians," in Proceedings of IEEE International Conference on Image Processing, Saint-Malo, France, September 26-29, Vol. 3, pp.1871-1874.

Farag, A. A., El-Baz, A., and Gimel'farb, G., 2006, "Precise segmentation of multi-modal images, "IEEE Transactions on Image Processing 15(4), 952-968.

Farag, A. A., El-Baz, A., Yuksel, S. E., El-Ghar, M. E. A., Eldiasty, T. A., and Ghoneim, M. A., 2006, "A framework for the detection of acute rejection with dynamic contrast enhanced magnetic resonance imaging," in IEEE International Symposium on Biomedical Imaging: From Nano to Macro (ISBI), Arlington, VA, April 6-9, pp. 418-421.

Gerig, G., Kikinis, R., Kuoni, W., van Schulthess, G. K., and Kubler, O., 1992, "Semiautomated ROI analysis in dynamic MRI studies: Part I: Image analysis tools for automatic correction of organ displacements," IEEE Transactions on Image Processing 11(2), 221-232.

Giele, E. L. W., 2002, Computer methods for semi-automatic MR renogram determination. PhD thesis, Department of Electrical Engineering, Eindhoven University of Technology, Eindhoven, The Netherlands.

El-Baz, A., Rachid, F., Yuksel, S. E., Farag, A. A., Miller, W., El-Ghar, M. A., and Eldiasty, T., 2006, "A new CAD system for the evaluation of kidney diseases using DCE-MRI," in Proceedings of International Conference on Medical Image Computing and Computer Assisted Intervention (MICCAI), Singapore, October 24-27, pp. $446-453$.

Rigg, K. M., 1995, “Renal transplantation: current status, complications and prevention,” Journal of Antimicrobial Chemotherapy 36, Suppl:B51-B57.

Schlesinger, M. I. and Hlavac, V., 2002, Ten Lectures on Statistical and Structural Pattern Recognition, Kluwer Academic, Norwell, MA

Sun, Y., Jolly, M., and Moura, J. M. F., 2004, "Integrated registration of dynamic renal perfusion MR images," in Proceedings of 2004 IEEE International Conference on Image Processing, Copenhagen, Denmark, October 1-6, pp. 1923-1926. 


\section{S.E. YUKSEL ET AL.}

Tsai, A., Yezzi, A., Wells, W., Tempany, C., Tucker, D., Fan, A., Grimson, E., and Willsky, A., 2003, "A shapebased approach to curve evolution for segmentation of medical imagery," IEEE Transactions on Medical Imaging 22(2), 137-154.

Witkin, A., Kass, M., and Terzopoulos, D., 1987, "Snakes: Active contour models," International Journal of Computer Vision 1, 321-331.

Yang, D., Ye, Q., Williams, M., Sun, Y., Hu, T. C. C., Williams, D. S., Moura, J. M. F., and Ho, C., 2001, "USPIO enhanced dynamic MRI: Evaluation of normal and transplanted rat kidneys," Magnetic Resonance in Medicine 46, 1152-1163.

Yuksel, S. E., 2005, Image processing methods for the detection of acute rejection after kidney transplantation. MS thesis, Department of Electrical and Computer Engineering, University of Louisville, Louisville, KY, December.

Yuksel, S. E., El-Baz, A., Farag, A. A., El-Ghar, M. E. A., Eldiasty, T. A., and Ghoneim, M. A., 2005, "Automatic detection of renal rejection after kidney transplantation," in Proceedings of Computer Assisted Radiology and Surgery (CARS), Berlin, Germany, June 22-25, pp. 773-778, June. 